\title{
X-exoplanets: an X-ray and EUV database for exoplanets
}

\author{
J. Sanz-Forcada ${ }^{1}$, D. García-Álvarez ${ }^{2,3}$, A. Velasco ${ }^{1,4}$, E. Solano ${ }^{1,4}$, \\ I. Ribas ${ }^{5}$, G. Micela ${ }^{6}$ and A. Pollock ${ }^{7}$ \\ ${ }^{1}$ Centro de Astrobiología, CSIC-INTA, European Space Astronomy Center \\ Apartado 78, E-28691 Villanueva de la Canada (Madrid), Spain \\ email: jsanz@cab.inta-csic.es \\ ${ }^{2}$ Instituto de Astrofísica de Canarias, Spain \\ ${ }^{3}$ Grantecan CALP, Spain \\ ${ }^{4}$ Spanish Virtual Observatory, Spain \\ ${ }^{5}$ Institut de Ciences de l'Espai (CSIC-IEEC), Spain \\ ${ }^{6}$ INAF- Osservatorio Astronomico di Palermo, Italy \\ ${ }^{7}$ XMM-Newton SOC, European Space Agency, ESAC, Spain
}

\begin{abstract}
Extreme Ultraviolet (EUV) and X-ray emission is of great importance in several phenomena related to the formation of planetary systems and the atmospheres of planets. The atmospheric composition, and the mass of an exoplanet, are partly dependent on the X-ray and EUV radiation received during the first stages of formation and even during main sequence of the star. Biological life developing on exoplanets would depend severely on the high energy radiation arriving from its parent star.

Here we present a database of the X-ray and EUV emission of all the stars currently known to host exoplanets. The archive is public and accessible through the Spanish Virtual Observatory (SVO). The database gives the user the option to download observed X-rays and EUV spectra. Synthetic spectra covering the spectral range 1-912 $\AA$ are also available (present day telescopes do not give access to the EUV range at $\lambda>180 \AA$ ). These spectra are created using coronal models after fitting observed spectra.
\end{abstract}

Keywords. astrobiology, (stars:) planetary systems, stars: coronae, stars: abundances, X-rays: stars, atomic data

\section{Introduction}

Since the discovery of the first transiting exoplanet, it has been proposed that high energy stellar radiation should have some influence on the atmosphere of the planets (e.g. Seager \& Sasselov 2000), in special the so called "hot Jupiters", planets with Jupiterlike mass at a short distance of the star. The effects of the high energy radiation (UV, EUV and X-rays) are important at different stages. First, during protoplanetary disc dissipation. Later, when the stellar rotation is fast and the planet keeps most of its original atmosphere, this radiation is believed to be a strong erosive agent for the atmosphere (e.g. Lammer et al. 2003). Finally, the developing of biological life in a planet will partly depend on the high energy (EUV and UV in special) radiation arriving to the surface.

During the evolution of a planet we have to consider also the evolution of its parent star. Once the protostellar disc dissipates, the rotation of the star will progressively slows down. The initial strong stellar rotation produces high coronal activity, with EUV and X-rays emission from the material heated at 1-30 MK. About 100 Myr after the stellar birth, the X-rays and EUV emission flux from the corona decreases and cools down, as 

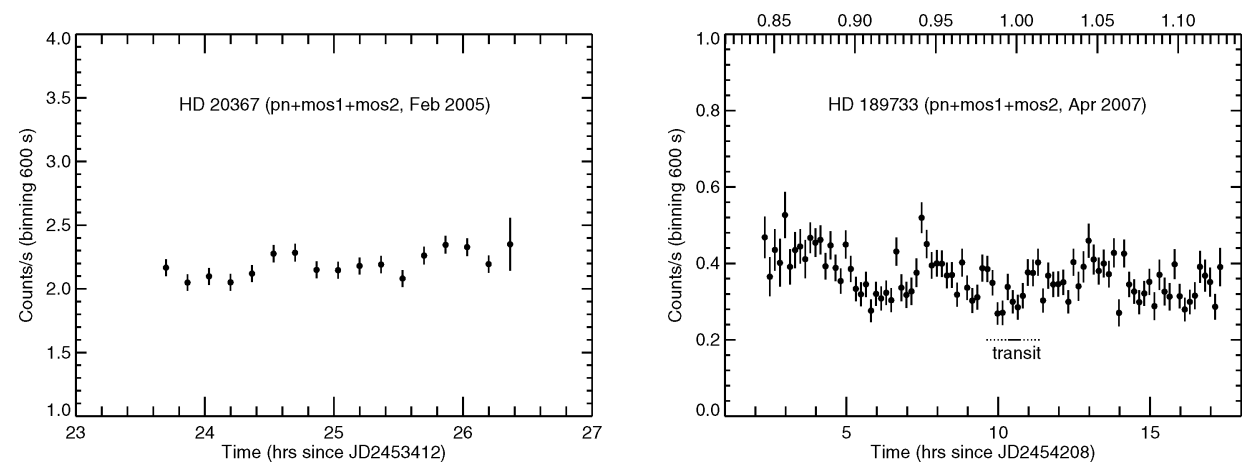

Figure 1. Two examples of X-ray light curves with some variability. Left: HD 20367, one of the most active stars in the sample. Right: HD 189733 hosts a transiting planet. The times for the total and partial (optical) transit are displayed, Upper axis show the orbital phase of the planet. Observations of evaporation signatures have been reported for this planet (Tinetti et al. 2007). High energy radiation is essential in this effect.

an effect of the slower rotation. The UV emission from the chromosphere and transition region follows the same pattern with age. It is possible to test the X-ray evolution with time by looking at the stellar clusters (Micela 2002). A direct application to the solar case was proposed by Ribas et al. (2005). A more detailed coronal model was employed by Cnossen et al. (2007) to evaluate the spectral energy distribution of $\kappa$ Cet, a proxy of the Sun at the time in which life developed on Earth.

In the last years there has been an increasing interest in the use of the high energy emission from the parent star to know the possible effects on the planet, such as vaporization or atmospheric "erosion" (Lammer et al. 2003; Baraffe et al. 2004; Erkaev et al. 2007; Penz \& Micela 2008; Penz et al. 2008; Cecchi-Pestellini et al. 2009; Lammer et al. 2009, Sanz-Forcada et al., submitted). These models are limited to the use of X-ray or EUV fluxes calculated in the whole band. The use of a spectral energy distribution, with the highest resolution achievable, would be prefered to know better the effects in the planet atmosphere. The access to high resolution spectra in this spectral range is, however, very limited. For X-rays there are currently two large telescopes (XMM-Newton and Chandra) providing the ranges $\sim 4-38 \AA$ (XMM-Newton/RGS) and $\sim 1.5-180 \AA$ (Chandra/HETGS and LETGS). Such spectra can only be obtained for the brightest
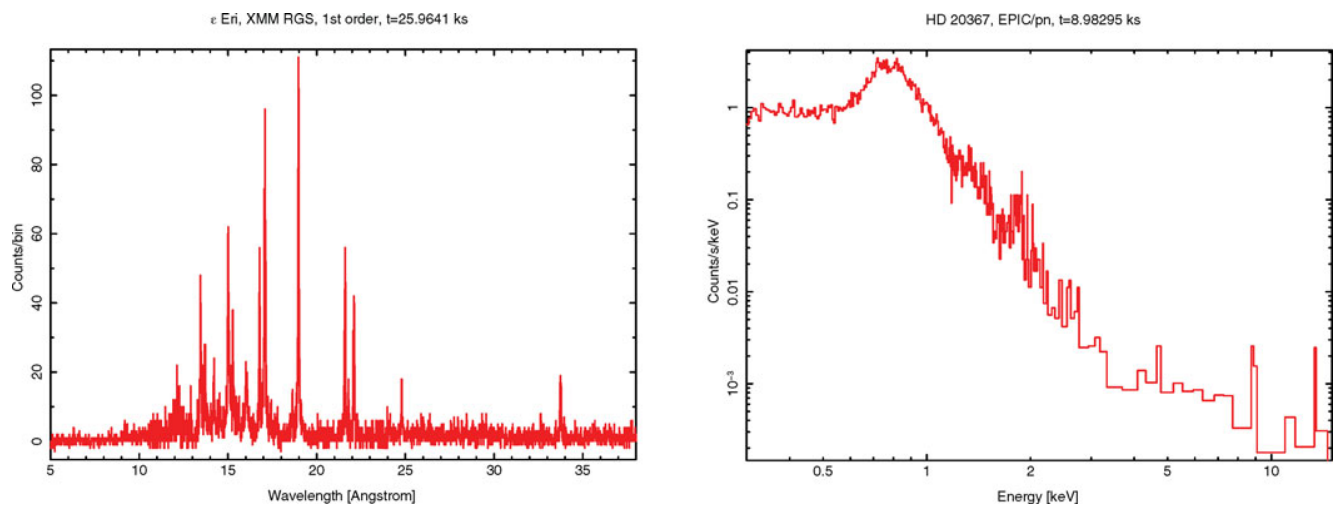

Figure 2. Real spectra observed with XMM-Newton. High resolution spectra (left) is only available for brightest stars. Most cases show, at most, a low-resolution spectrum (right). 
Table 1. XMM-Newton flux of stars with exoplanets $(0.12-2.48 \mathrm{keV})^{1}$

\begin{tabular}{|c|c|c|c|c|c|c|c|}
\hline Planet name & $\begin{array}{l}\text { Spectral type } \\
\text { (star) }\end{array}$ & $\begin{array}{c}\text { Stellar distance } \\
(\mathrm{pc})\end{array}$ & $\begin{array}{c}\log L_{\mathrm{X}} \\
\left(\operatorname{erg~s}^{-1}\right)\end{array}$ & $\mathrm{S} / \mathrm{N}\left(L_{\mathrm{X}}\right)$ & $\begin{array}{c}m \sin i \\
\left(\mathrm{~m}_{J}\right)\end{array}$ & $\begin{array}{c}a_{p} \\
\text { (a.u.) }\end{array}$ & $\begin{array}{c}P_{\text {orb }} \\
(\mathrm{d})\end{array}$ \\
\hline 14 Her b & $\mathrm{K} 0 \mathrm{~V}$ & $18.10 \pm 0.19$ & 26.92 & 4.9 & 4.64 & 2.77 & 1773.4 \\
\hline $47 \mathrm{Uma}$ b & G0V & $13.97 \pm 0.13$ & $<25.45$ & $\ldots$ & 2.60 & 2.11 & 1083.2 \\
\hline $47 \mathrm{Uma} \mathrm{c}$ & " & " & " & $"$ & 0.46 & 3.39 & 2190.01 \\
\hline$\beta \mathrm{Pic} \mathrm{b}$ & $\mathrm{A} 6 \mathrm{~V}$ & $19.30 \pm 0.19$ & 25.63 & 5.75 & 8.00 & 8.00 & 6000 \\
\hline $\mathrm{Cha} \mathrm{H} \alpha 8 \mathrm{~b}$ & M6.5 & 160 & $<27.87$ & & 18.00 & 1.00 & 1590.9 \\
\hline$\epsilon$ Eridani b & $\mathrm{K} 2 \mathrm{~V}$ & $3.20 \pm 0.01$ & 28.20 & 297 & 1.55 & 3.39 & 2502 \\
\hline GQ Lup b & $\mathrm{K} 7 \mathrm{eV}$ & 140 & 29.45 & 32 & 21.50 & 103 & \\
\hline HD $20367 \mathrm{~b}$ & G0 & $27.00 \pm 0.79$ & 29.30 & 140 & 1.07 & 1.25 & 500 \\
\hline HD $46375 \mathrm{~b}$ & K1IV & $33.40 \pm 1.19$ & 27.16 & 7.3 & 0.25 & 0.04 & 3.02 \\
\hline HD $49674 \mathrm{~b}$ & $\mathrm{G} 5 \mathrm{~V}$ & $40.70 \pm 1.89$ & 27.41 & 6.5 & 0.12 & 0.06 & 4.94 \\
\hline HD $50554 \mathrm{~b}$ & $\mathrm{~F} 8$ & $31.03 \pm 0.97$ & $<26.59$ & $\ldots$ & 4.90 & 2.38 & 1279 \\
\hline HD $62509 \mathrm{~b}$ & K0IIIb & $10.34 \pm 0.09$ & 27.13 & 34 & 2.90 & 1.69 & 589.64 \\
\hline HD $70642 \mathrm{~b}$ & G5IV-V & $29.00 \pm 0.50$ & 26.39 & 4.2 & 2.00 & 3.30 & 2231 \\
\hline HD $75289 \mathrm{~b}$ & G0V & $28.94 \pm 0.47$ & $<26.16$ & $\ldots$ & 0.42 & 0.05 & 3.51 \\
\hline HD $108147 \mathrm{~b}$ & F8/G0V & $38.57 \pm 1.03$ & 27.39 & 4.2 & 0.26 & 0.10 & 10.90 \\
\hline HD $114762 \mathrm{~b}$ & F9V & $39.46 \pm 2.37$ & $<26.61$ & & 11.02 & 0.30 & 83.89 \\
\hline HD $130322 \mathrm{~b}$ & $\mathrm{~K} 0 \mathrm{~V}$ & $30.00 \pm 1.34$ & 27.26 & 7.7 & 1.08 & 0.09 & 10.72 \\
\hline HD $187123 \mathrm{~b}$ & G5 & $50.00 \pm 1.63$ & $<26.80$ & $\ldots$ & 0.52 & 0.04 & 3.10 \\
\hline HD $187123 \mathrm{c}$ & " & $"$ & " & $"$ & 1.99 & 4.89 & 3810 \\
\hline HD $189733 \mathrm{~b}$ & $\mathrm{~K} 1-\mathrm{K} 2$ & $19.30 \pm 0.32$ & 28.18 & 93 & 1.13 & 0.03 & 2.22 \\
\hline HD $190360 \mathrm{~b}$ & G6IV & $15.89 \pm 0.16$ & $<26.38$ & $\ldots$ & 1.50 & 3.92 & 2891 \\
\hline HD $190360 \mathrm{c}$ & 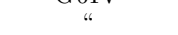 & " & 4 & " & 0.06 & 0.13 & 17.10 \\
\hline HD $195019 \mathrm{~b}$ & G3IV-V & $37.36 \pm 1.24$ & $<26.52$ & $\ldots$ & 3.70 & 0.14 & 18.20 \\
\hline HD 209458 b & G0V & $47.00 \pm 2.22$ & $<26.12$ & $\ldots$ & 0.69 & 0.05 & 3.52 \\
\hline HD $216435 \mathrm{~b}$ & G0V & $33.30 \pm 0.81$ & 27.74 & 12 & 1.26 & 2.56 & 1311 \\
\hline HD $216437 \mathrm{~b}$ & G4IV-V & $26.50 \pm 0.41$ & 26.62 & 3.9 & 2.10 & 2.70 & 1294 \\
\hline HD $217107 \mathrm{~b}$ & G8IV & $19.72 \pm 0.29$ & $<25.30$ & $\ldots$ & 1.33 & 0.07 & 7.13 \\
\hline HD $217107 \mathrm{c}$ & " & " & " & $"$ & 2.49 & 5.27 & 4210 \\
\hline HD $330075 \mathrm{~b}$ & G5 & $50.20 \pm 3.75$ & 26.51 & 3.1 & 0.76 & 0.04 & 3.37 \\
\hline NGC $24233 \mathrm{~b}$ & & 766 & $<29.47$ & $\ldots$ & 10.60 & 2.10 & 714.3 \\
\hline$\tau$ Boo b & $\mathrm{F} 7 \mathrm{~V}$ & $15.60 \pm 0.17$ & 28.94 & 317 & 3.90 & 0.05 & 3.31 \\
\hline $\mathrm{VB} 10 \mathrm{~b}$ & $\mathrm{M} 8 \mathrm{~V}$ & $6.09 \pm 0.13$ & 25.83 & 20 & 6.40 & 0.36 & 271.56 \\
\hline
\end{tabular}

Notes: ${ }^{1}$ Planet data taken from The Extrasolar Planets Encyclopedia (http://exoplanet.eu)

X-ray sources. Among the planet-bearing stars only two have good spectra in this range, $\epsilon$ Eri and $\tau$ Boo (Sanz-Forcada et al. 2004; Maggio et al. 2009), with a few more cases with lower quality spectra, such as HD 20367 (Table 1). In most cases we will be limited to low-resolution spectra (range $\sim 1-125 \AA$ ), and even upper limits of the flux for the lower activity stars. The situation is even worse in the EUV range $(\sim 100-900 \AA)$, where no current instrumentation can observe, except for the small range covered by X-rays telescopes. The Extreme Ultraviolet Explorer (EUVE) observed a few dozens of cool stars until the year 2000, most of them at close distances. The main limitation of that mission was the absorption by the hydrogen in the interstellar medium (ISM), that becomes stronger for longer wavelengths, up to $912 \AA$. Only one star with a known exoplanet was observed with EUVE, $\epsilon$ Eri (Sanz-Forcada et al. 2003a). The close distance of the star resulted in a good spectrum up to $\sim 400 \AA$ (Fig. 4).

In view of this situation we have constructed a database ("X-exoplanets") of real and synthetic spectra of stars with exoplanets. Since the real data that can be used in the models is very scant, we have created a set of high-resolution spectra constructed using coronal models. These models have been calculated using the real spectra available, and have been tested with $\epsilon$ Eri, the only case in which a good EUV spectrum is available. Most emission in both, the X-rays and EUV ranges, is produced in the corona, except for a few chromospheric and transition region lines. The database covers all the planet-bearing stars that have been observed with XMM-Newton or Chandra, and will be updated regularly as new data are incorporated. In the next lines we explain in detail how the 

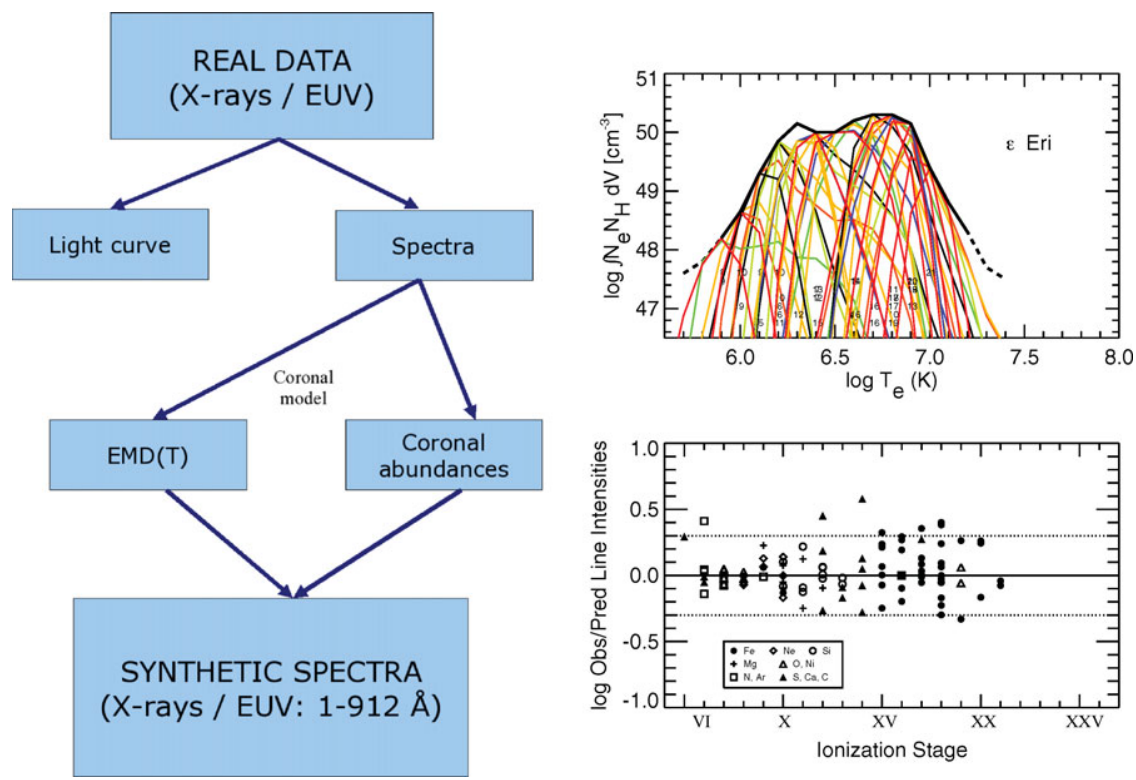

Figure 3. Left: schematic view of the technique employed to create the synthetic spectra from real data. Right: the high resolution data allow us to do a detailed reconstruction of the emission measure distribution. Synthetic spectra can be provided by use of this coronal model, in combination with the coronal abundances, also calculated during the process.

synthetic spectra are constructed, and what are the products that can be retrieved from $\mathrm{X}$-exoplanets. The database can be accessed through the Spanish Virtual Observatory at the address http://sdc.cab.inta-csic.es/xexoplanets/

\section{The database}

The X-exoplanets database has the purpose to reduce all XMM-Newton and Chandra (eventually also EUVE) public data of stars with exoplanets reported, and to make these products available to the general public. We provide the light curves in different time bins (Fig. 1), and the low and high resolution spectra of the different instruments when available (Fig. 2). Some sources have not been detected, so circular regions have been selected at the expected position of the source, corrected from proper motion for the observing date; for those sources a light curve will be provided, and only an upper limit of the X-rays luminosity is included. For targets with enough statistics, the low resolution spectra have been fitted using rather simple coronal models, consisting on 1 to 3 temperature components, and a limited sample of element abundances, depending on the quality of the data (most sources can be well fitted with just 1 component). The spectra and necessary files are also provided, together with plotted spectra and residuals of the fit, and the coronal model used in the fit. For the few cases with high-resolution spectra, a detailed coronal model has been calculated using a line-based method, as described in Sanz-Forcada et al. (2003b), and references therein. A whole set of element abundances, and a coronal model with a $\Delta T=0.1$ resolution are used in the fit.

The positions of the targets have been carefully calculated to improve former results. A comparison with past works show some inconsistencies with our X-ray fluxes $\left(L_{\mathrm{X}}\right)$. Since this quantity has little dependence on the exact parameters introduced in the fitting model, we attribute the discrepancy to wrong identifications of the sources. The case 

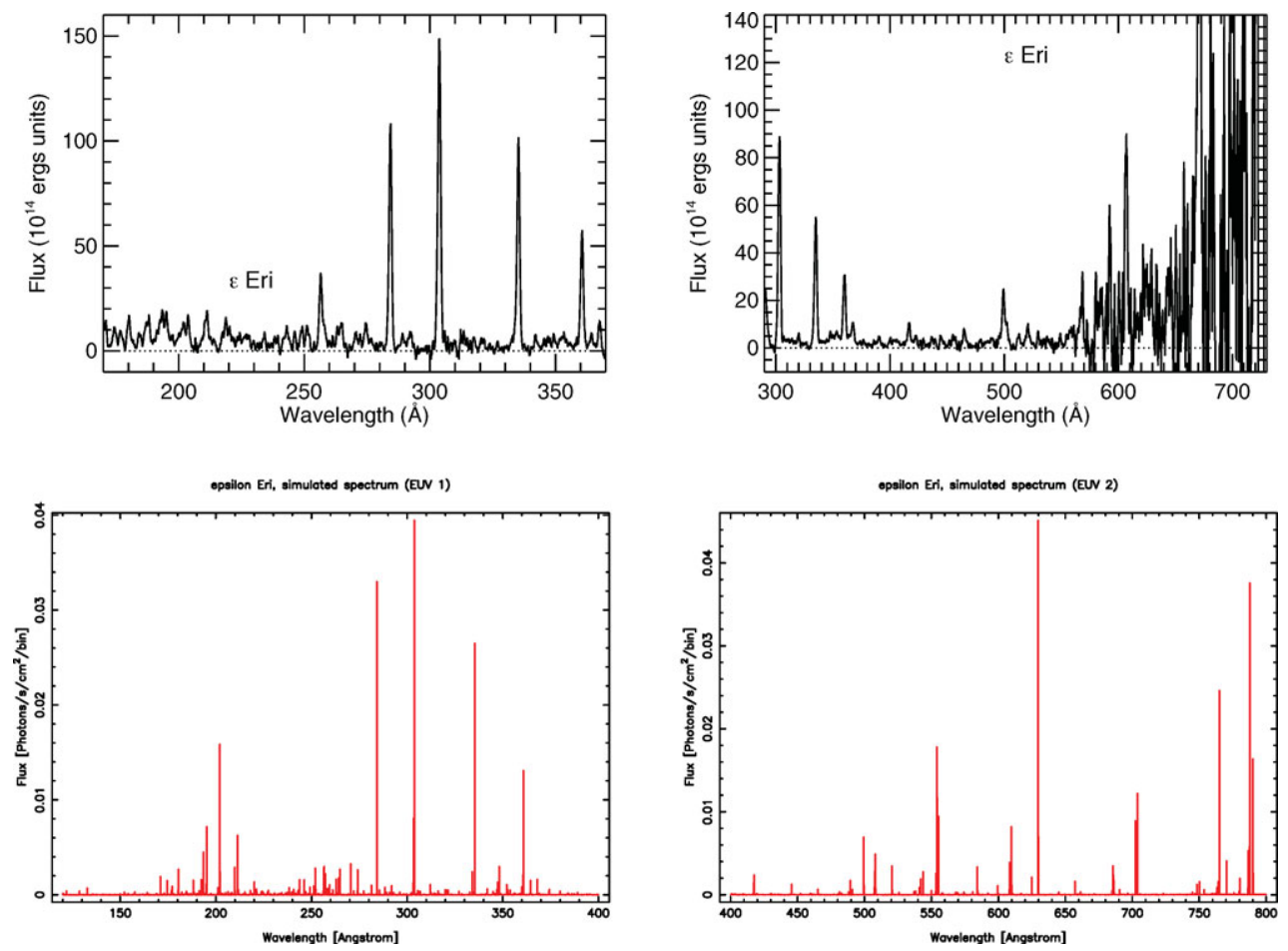

Figure 4. Real spectra (upper figures) of $\epsilon$ Eri observed with EUVE, in flux units. Note that the absorption in the interstellar medium allows very poor observations at longest EUV wavelengths. Synthetic spectra (lower figures) constructed with the coronal model successfully reproduces most spectral features of the real data.

of $47 \mathrm{UMa}$ is particularly interesting. Kashyap et al. (2008) calculate in XMM-Newton $\log L_{\mathrm{X}}=27.13 \mathrm{erg} \mathrm{s}^{-1}$. However the EPIC-pn image reveals two sources in the area of 47 UMa. The star itself $\left(\alpha=10: 59: 28.0, \delta=40: 25: 46, \log L_{\mathrm{X}}<25.45 \mathrm{erg} \mathrm{s}^{-1}\right)$ and a brighter source at $\alpha=10: 59: 26.7, \delta=40: 26: 04$, with a spectrum that resembles that of an AGN with $\mathrm{z} \sim 0.2$ and $\log L_{\mathrm{X}} \sim 43 \operatorname{erg~s}^{-1}$ (G. Miniutti, private comm.). For the star HD 209458, that has been used in several studies because of its transiting planet, only an upper limit of $\log L_{\mathrm{X}}=26.12 \mathrm{erg} \mathrm{s}^{-1}$ could be calculated, while Kashyap et al. (2008) cite $\log L_{\mathrm{X}}=27.02 \mathrm{erg} \mathrm{s}^{-1}$, probably confused with one of the nearby sources, well identified by us with 2MASS counterparts. This problem is even worse for ROSAT detections, given the poor resolution of ROSAT/PSPC.

\section{Synthetic spectra}

In order to have a better resolution spectra that can be used in the planetary atmospheric models, we have used our calculated coronal models to synthesize such spectra, using the atomic database APED (Smith et al. 2001). The method is described in Fig. 3. The central idea is that the emission in both, X-rays and EUV ranges, is originated in the corona of the star. The knowledge of the coronal model (basically the emission measure distribution, EMD, Fig. 3) and coronal abundances allows us to synthesize the whole spectral range, even if we have observations only in X-rays. The EMD is the distribution of coronal mass with temperature. The best is the model, the better will be the synthetic spectra; Low spectral resolution results only in a global fit to the spectrum and 
just 1-3 components of the EMD. But the high resolution data allow us to do a detailed reconstruction of the EMD, now calculated by comparing observed line fluxes with the fluxes predicted by the combination of the atomic data and different trial EMDs (see Sanz-Forcada et al. 2003b, for further details).

The atomic models are quite accurate for short wavelengths, but they have not been so well tested at longer wavelengths $(\gtrsim 500 \AA)$. Besides, a few lines originated at lower temperatures (in the chromosphere or the transition region) are also present in the EUV range (most notably the He II $304 \AA$ line). For a few cases there are UV observations and the coronal model can be extended to lower temperatures to reproduce these lines. For other targets we are using scaling laws from the coronal EMD, based on past observations available in Sanz-Forcada et al. (2003a). In order to test the validity of the method we have used $\epsilon$ Eri, the case with better spectral coverage. The coronal model of this star has been calculated using Chandra and EUVE high resolution spectra (see also XMM-Newton RGS spectrum in Fig. 2). International Ultraviolet Explorer (IUE) spectra extends the EMD to the transition region and the chromosphere, so a few "cooler" lines produced below $912 \AA$ are properly modeled (Sanz-Forcada et al. 2003a, 2004). The results obtained are very promising (Fig. 4), with the models reproducing the main lines at $\lesssim 400 \AA$ accurately. For longer wavelengths the synthetic spectra are still valid, but the quality of the real spectra falls at that range due to ISM absorption, and atomic models have not been tested so much as for shorter wavelengths.

This technique has been successfully applied to the star $\kappa$ Cet (Cnossen et al. 2007) in order to see how the early Earth atmosphere would absorb the high energy emission from the star at the moment when life started on Earth. The use of this technique is particularly promising for transiting planets, in which some features, triggered as an effect of the incoming high-energy emission, might be observable in the transmitted spectrum of the planet.

\section{References}

Baraffe, I., Selsis, F., Chabrier, G., et al. 2004, A\&A(Letters), 419, L13

Cecchi-Pestellini, C., Ciaravella, A., Micela, G., \& Penz, T. 2009, A\&A, 496, 863

Cnossen, I., Sanz-Forcada, J., Favata, F., et al. 2007, Journal of Geoph. Res. (Planets), 112, E02008

Erkaev, N. V., Kulikov, Y. N., Lammer, H., et al. 2007, A\&A, 472, 329

Kashyap, V. L., Drake, J. J., \& Saar, S. H. 2008, ApJ, 687, 1339

Lammer, H., Bredehöft, J. H., Coustenis, A., et al. 2009, A\&GAR, 17, 181

Lammer, H., Selsis, F., Ribas, I., et al. 2003, ApJ(Letters), 598, L121

Maggio, A., Sanz-Forcada, J., \& Scelsi, L. 2009, in Proc. of the IAU Symposium No. 265, Chemical abundances in the Universe: connecting first stars to planets, ed. K. Cunha, M. Spite, \& B. Barbury, in press

Micela, G. 2002, in ASP Conf. Series, Vol. 269, The Evolving Sun and its Influence on Planetary Environments, ed. B. Montesinos, A. Gimenez, \& E. F. Guinan, p. 107

Penz, T. \& Micela, G. 2008, A\&A, 479, 579

Penz, T., Micela, G., \& Lammer, H. 2008, $A \& A$, 477, 309

Ribas, I., Guinan, E. F., Güdel, M., \& Audard, M. 2005, ApJ, 622, 680

Sanz-Forcada, J., Brickhouse, N. S., \& Dupree, A. K. 2003a, ApJS, 145, 147

Sanz-Forcada, J., Favata, F., \& Micela, G. 2004, A\& A, 416, 281

Sanz-Forcada, J., Maggio, A., \& Micela, G. 2003b, A\&AA, 408, 1087

Seager, S. \& Sasselov, D. D. 2000, ApJ, 537, 916

Smith, R. K., Brickhouse, N. S., Liedahl, D. A., \& Raymond, J. C. 2001, ApJ(Letters), 556, L91

Tinetti, G., Vidal-Madjar, A., Liang, M.-C., et al. 2007, Nature, 448, 169 